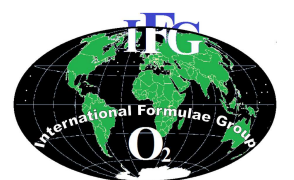

Available online at http://ajol.info/index.php/ijbcs

Int. J. Biol. Chem. Sci. 8(4): 1697-1709, August 2014

International Journal

of Biological and

Chemical Sciences

ISSN 1997-342X (Online), ISSN 1991-8631 (Print)

Original Paper

http://indexmedicus.afro.who.int

\title{
Evaluation et amélioration du comportement de Atriplex lentiformis (Torr.) S. Watson en milieux salés au Sénégal
}

\author{
Elhadji FAYE ${ }^{1 *}$, Mouhameth CAMARA ${ }^{1}$, Mamoudou Abdoul TOURE ${ }^{2}$ et \\ Abdou MBAYE ${ }^{2}$ \\ ${ }^{1}$ Université de Thiès, Institut Supérieur de Formation Agricole et Rurale, BP 54 Bambey, Sénégal. \\ ${ }^{2}$ Institut Sénégalais de Recherches Agricoles, Centre National de Recherches Forestières, BP 2312 Dakar- \\ Hann, Sénégal. \\ *Auteur correspondant ; E-mail : elhadji.faye@univ-thies.sn
}

\section{RESUME}

La salinisation des sols constitue l'une des principales menaces à la productivité agricole dans les zones estuariennes du Sénégal. La mycorhization pourrait améliorer l'efficacité de la méthode biologique de désalinisation des sols. Atriplex lentiformis associé ou non au champignon Rhizophagus irregulare a été observé en milieu réel, et en serre dans un dispositif bi-factoriel (mycorhization et salinité) en blocs complets randomisés avec 3 répétitions. Les variables mesurées sont la hauteur, le diamètre, la litière, la teneur en eau, en sodium des plants, la salinité et l'acidité du sol. Les résultats montrent un taux de mortalité supérieur à $90 \%$ en milieu réel. Les individus plantés sur ados ont développé un enracinement superficiel alors que les semis naturels ont présenté un système racinaire pivotant et profond. La mycorhization a entraîné une réduction de la mortalité de 5,56\%, une importante persistance des feuilles, une teneur en eau plus élevée dans les tiges et racines que dans les feuilles et enfin un stockage de sodium plus élevé dans les feuilles. La dose de sel 186 g. $1^{-1}$ a été létale pour Atriplex lentiformis, mycorhizé ou non. La symbiose endomycorhizienne améliore ainsi le comportement de Atriplex lentiformis en milieu salé.

(C) 2014 International Formulae Group. All rights reserved.

Mots clés : Atriplex lentiformis, mycorhization, salinité, stress, Sénégal.

\section{INTRODUCTION}

Dans les zones arides, en Australie comme en Afrique du nord par exemple, les espèces de la famille des Chenopodiaceae sont largement utilisées dans la réhabilitation et la mise en valeur des terres arides très hostiles (Franclet et Le Houérou, 1971; Le Houérou, 1992 ; Orev, 1987 ; Rusbridge et al., 1996 ). Atriplex est un genre de la famille des Chenopodiaceae qui comporte des espèces connues pour leur résistance à la sécheresse et à la salinité. Franclet et Le Houérou (1971) ont listé 417 espèces du genre Atriplex qui comptent parmi les plantes utiles les mieux équipées pour lutter contre la salure. Atriplex lentiformis (Torr.) S. Watson, satisferait $a$ priori aux critères de choix d'espèces de reboisement des sols salés de Ndiaffate, dans les basses vallées du fleuve Saloum, au Sénégal. Ces critères incluent la résistance au sel, la couverture du sol et les produits secondaires fournis tels que le bois, les feuilles, etc.

Ainsi, dans le cadre du Projet de Restauration Agronomique des Sols Salés (PRASS), les diguettes utilisées dans la mise 
en place des périmètres de récupération des sols à Ndiaffate, ont été plantées de $A$. lentiformis (Torr.) S. Watson. Cette plantation avait pour objectif de stabiliser ces diguettes. Mais le comportement en général mitigé des Atriplex a été signalé par de nombreux auteurs tels que Chatterton et McKell (1969) et Olivares et Johnson (1978). Par la suite, très peu d'études ont été consacrées à $A$. lentiformis en milieux salés. Or les sols salés représentent 1700000 ha au Sénégal selon LADA (2003). Aussi, pour mieux promouvoir la récupération et la valorisation de ce type de sol, le présent travail cherche à améliorer, grâce à la mycorhization, le comportement de A. lentiformis face au stress salin.

\section{MATERIEL ET METHODES Evaluation in situ}

Les plants de A. lentiformis ont été installés sur des ados à sommets non amortis en 1999. Ces ados sont le sommet des diguettes périmétrales des 20 parcelles élémentaires composant le dispositif expérimental de Ndiaffate situé à $20 \mathrm{~km}$ au sud de la région de Kaolack au centre du Sénégal (1404'22,37" Nord ; 16²10'56,83" Ouest). C'est un dispositif en blocs complets randomisés répartis le long de la toposéquence et destinés à la restauration agronomique des terres salées. Des regarnis ont été réalisés en 2000, 2001 et 2002.

Au cours du mois de juin 2003, ces plantations de A. lentiformis ont été inventoriées. Ensuite, les deux plus grands individus survivants et les semis naturels ont été ciblés pour étudier le système racinaire et les propriétés chimiques de la rhizosphère de A. lentiformis. Pour l'étude du profil racinaire, une fosse de $1 \mathrm{~m}$ de profondeur a été ouverte à $10 \mathrm{~cm}$ de chaque arbre. Dans chaque fosse, un cadre en bois de $1 \mathrm{~m}^{2}$ constitué de 100 mailles de $10 \mathrm{~cm}$ de côté a été utilisé pour repérer les racines. L'axe des ordonnées correspond au centre de la grille dans le prolongement du collet. L'axe des abscisses correspond au plan horizontal de la surface du sol. Sur ce plan, toute racine qui affleure a été mesurée (diamètre) et les coordonnées ont été notées.
Ces données ont permis de tracer le profil racinaire des arbres.

Des échantillons de sols ont été prélevés dans les horizons 0-30, 30-60 et 60$90 \mathrm{~cm}$ autour d'individus situés en bas et au milieu de la toposéquence puis analysés au laboratoire de chimie de l'Institut de Recherche pour le Développement (IRD) de Hann Dakar certifié ISO9001. Les variables étudiées ont été le $\mathrm{pH}$ et la salinité.

\section{Evaluation ex situ \\ Matériel végétal}

Le matériel végétal utilisé a été constitué de jeunes plants de A. lentiformis produits en pépinière à la station de Ndiaffate et âgés de 9 mois. Les semences ont été récoltées sur ce site. Le semis a été effectué sans prétraitement dans des bacs de germination dont le substrat était constitué d'un sol de Ndiaffate stérilisé (à l'étuve à 120 ${ }^{\circ} \mathrm{C}$ pendant 48 heures) et enrichi en vermiculite. Un mois après la germination, les jeunes plants ont été repiqués dans 320 gaines de polyéthylène de $25 \mathrm{~cm}$ de haut sur $5 \mathrm{~cm}$ de large. Ces gaines ont été remplies de sable dunaire stérilisé à $120^{\circ} \mathrm{C}$ pendant 48 heures.

La moitié des plants âgés d'un mois a été inoculée avec une souche de champignon endomycorhizien Rhizophagus irregulare. L'autre moitié non inoculée a servi de plants témoins. L'arrosage a été effectué matin et soir en raison de 0,05 litre par plants et par jour avec l'eau de robinet alcaline, caractérisée par un $\mathrm{pH}$ de 8,17, une conductivité électrique $(\mathrm{CE})$ à $20^{\circ} \mathrm{C}$ de 1,61 $\mathrm{mS} . \mathrm{cm}^{-1}$, une concentration en $\mathrm{CO}^{2-}$ de 230 mg. $1^{-1}$, en $\mathrm{Cl}^{-}$de $359 \mathrm{mg} . \mathrm{l}^{-1}$ et en $\mathrm{SO}^{2-}$ de $57,2 \mathrm{mg} . \mathrm{l}^{-1}$ plus faible que celle de Ndiaffate. A partir du sixième mois, les plants ont été arrosés une fois par jour. Ces plants de $20 \mathrm{~cm}$ de haut ont été transplantés dans des gaines plus grandes de $30 \mathrm{~cm}$ x $22 \mathrm{~cm}$ remplies d'un mélange stérilisé et plus riche, de sable de Mbao (2/3) et de terreau (1/3). Les caractéristiques granulométriques de ce mélange (Roussel, 1995) sont présentées dans le Tableau 1. 


\section{Dispositif expérimental}

Les plants préalablement cultivés pendant 9 mois sur un milieu non salé ont été installés en gaines dans un dispositif expérimental de stress salin afin d'étudier les effets améliorants de la mycorhization sur le comportement des jeunes plants. La vérification de l'effectivité de la symbiose mycorhizienne a été faite avant la mise en place du dispositif expérimental. L'observation de la mycorhization arbusculaire a été effectuée après le traitement des racines par la technique de Philips et Haymans (1970).

Le dispositif mis en place est factoriel à 2 facteurs en blocs complets randomisés :

- le facteur 1 est l'inoculation mycorhizienne avec deux modalités (absence et présence) ;

- le facteur 2 est la salinité avec 6 niveaux 0 ; 2,$92 ; 5,84 ; 8,76 ; 11,68 ; 23,36$ g. $^{-1}$ (respectivement notés S0, S1, S2, S3, S4, S5) appliqués pendant 2 mois et demi ; à partir du 11 juillet 2002, de nouvelles doses ont été testées pendant 21 jours, ces doses, 8 fois plus élevées que les premières, sont nommées respectivement $\mathrm{S} 0, \mathrm{~S} 5, \mathrm{~S} 6, \mathrm{~S} 7, \mathrm{~S} 8, \mathrm{~S} 9$ (0 ; 23,36 46,72; 93,44; 186,88 g..$^{-1}$ ).

La combinaison des modalités des facteurs 1 et 2 donne 12 traitements étudiés. Pour chaque traitement, l'unité expérimentale a été constituée de 6 plants.

Les apports de sel ont été faits progressivement afin d'éviter les chocs osmotiques aux jeunes plants. Ainsi, la dose initiale apportée a été de $25 \mathrm{mM}\left(1,46\right.$ g. $\left.\mathrm{l}^{-1}\right)$ prise comme seuil de tolérance des espèces non halophytes. Cette dose a été multipliée par deux, tous les deux jours jusqu'à l'obtention des doses retenues pour l'essai, suivant la suite géométrique $\left(\mathrm{U}_{\mathrm{n}}\right)$ de raison 2 et de premier terme $1,46 \mathrm{~g} . \mathrm{l}^{-1}$ représentée par la formule : $U_{n}=U_{0} \times 2^{n}$. (où $U_{n}$ représente la dose du $\mathrm{n}^{\mathrm{e}}$ jour $; \mathrm{U}_{0}=1,46 \mathrm{~g} . \mathrm{l}^{-1} ; 2$ représente la raison).

Au cours de l'essai, les plants ont été arrosés une fois par jour avec la solution saline à raison de $50 \mathrm{ml} \mathrm{d}$ 'eau par plant. Les variables chimiques $(\mathrm{pH}$, Conductivité électrique de l'extrait de pâte saturée (CE à 20
${ }^{\circ} \mathrm{C}$ ), teneurs en sodium, calcium et potassium des feuilles et racines) et biologiques (croissance et production : hauteur, diamètre, litière foliaire) des plants ont été suivies. Au total, 648 échantillons de feuilles, tiges et racines en raison de 6 échantillons par traitement pour l'évaluation de la teneur en eau, 72 échantillons de feuilles et racines en raison de 2 par traitement pour la teneur en sodium et 144 échantillons de litières en raison de 4 par traitement, ont été collectés. La phytomasse récoltée a été séchée à l'étuve à $70{ }^{\circ} \mathrm{C}$ pendant 48 heures. L'analyse chimique des 72 échantillons a été faite au laboratoire de chimie de l'IRD Dakar.

\section{Traitement statistique des données}

Les données recueillies ont été soumises à des analyses de variance avec le logiciel SAS version 8.2.

\section{RESULTATS} in situ :

Comportement de Atriplex lentiformis

Taux de survie et régénération naturelle

Les premières plantations ont été faites en 1999 suivies de regarnis en 2000 et 2001 (Tableau 2). Le taux de survie de $A$. lentiformis a diminué au fil du temps. Il a été faible pour les plants de $2001(7 \%)$ et 2000 (5\%). Pour les plantations de 1999 , le taux de survie des arbres a été inférieur à $1 \%$ (Tableau 2).

Caractérisations des pieds de $A$. lentfiormis

\section{Profils aériens des pieds étudiés}

Les arbres 1 et 2 sont respectivement âgés de 3 et 2 ans (2000 et 2001), hauts de 173 et $126 \mathrm{~cm}$, gros de 10 et $6 \mathrm{~cm}$ (Photo $1 \mathrm{a}$ et b).

\section{Profils souterrains des pieds étudiés}

Deux faits majeurs ont été observés dans les profils 1 et 2 (Figure 1 a et $b$ ) :

- les racines fines $(<1 \mathrm{~mm}$ de diamètre) colonisent le profil de 0 à $100 \mathrm{~cm}$ pour l'arbre 2 et jusqu'à $90 \mathrm{~cm}$ pour l'arbre 1 . La distribution de ces racines a été plus homogène chez l'arbre 2 que chez l'arbre 1. 
- les racines les plus grosses $(>1 \mathrm{~mm}$ et $>1$ $\mathrm{cm})$ ont été plutôt localisées dans les 30 premiers $\mathrm{cm}$ du sol et autour du houppier. Ce type d'enracinement superficiel ou traçant pour les arbres de plus de 2 ans, montre l'absence d'un pivot puissant orienté vers la profondeur chez les pieds plantés sur des ados.

L'architecture racinaire des jeunes plants a été examinée à partir des semis naturels âgés d'une année (Photo $2 \mathrm{a}$ et b) et rencontrés en bas de toposéquence. Elle montre un enracinement pivotant (Photo 2d). Le pivot n'est pas ramifié, et descend verticalement en profondeur. Il se prolonge à plus de $50 \mathrm{~cm}$ de profondeur contournant parfois certains obstacles par un angle de $90^{\circ}$. Cependant, la Photo 2c montre une racine secondaire traçante localisée dans les 15 premiers $\mathrm{cm}$ du profil et longue de plus de 70 $\mathrm{cm}$ (ligne noire).

\section{Profils chimiques des pieds étudiés}

$\mathrm{Au}$ pied des arbres, l'analyse de l'acidité a montré que le sol était acide avec un $\mathrm{pH}>5$ entre 0 et $90 \mathrm{~cm}$ de profondeur. Cette acidité a été plus faible chez l'arbre 1 que chez l'arbre 2 dans les horizons $0-30 \mathrm{~cm}$ et $60-90 \mathrm{~cm}$. Par contre, à l'horizon $30-60 \mathrm{~cm}$, l'acidité a été plus basse autour des semis naturels. Cela semble favoriser leur émergence en bas de toposéquence où 7 jeunes régénérations séminales ont été inventoriées non loin du lit du bras du fleuve (Figure 2).

La salinité a été plus élevée autour de l'arbre 2 comparé à l'arbre 1 sur tout le profil pédologique. Les horizons superficiel (0-30 $\mathrm{cm})$ et profond $(60-90 \mathrm{~cm})$ ont été plus salés que l'horizon intermédiaire $(30-60 \mathrm{~cm})$. Elle a aussi été plus élevée dans l'horizon 30-60 cm autour de la régénération séminale comparée à l'arbre 1 (Figure 3).

\section{Effets de la mycorhization sur le comportement ex situ de Atriplex lentiformis \\ Salinité et mortalité des plants}

Le sol de l'essai commence à être salé (salinité du sol > 2,44 g.l-1) seulement à partir de la dose seuil $\mathrm{S}_{16}$ ou 46,72 g.l-1 de l'eau d'arrosage. A partir de ce seuil $\mathrm{S}_{16}$, la salinité du sol a toujours été moins élevée dans les pots mycorhizés comparés à ceux non mycorhizés. Logiquement, les plants mycorhizés devraient utiliser plus de sel d'arrosage pour justifier cette différence de salinité résiduelle du sol. A ce seuil de salinité, aucune mortalité n'a été constatée chez les plants mycorhizés alors que ceux non mycorhizés en présentent déjà $5,56 \%$. Cet effet positif de la mycorhization semble s'estomper à partir de la dose d'arrosage $S_{32}$ ou 93,44 g.l-1 presque le triple de l'eau de mer (Tableau 3).

\section{Croissance en hauteur}

Les résultats de la Figure 4 montre que la hauteur n'a pas été influencée par la présence de mycorhizes pour les $\mathrm{S}_{0}, \mathrm{~S}_{8}, \mathrm{~S}_{16}$ et $\mathrm{S}_{32}$. Aucun plan n'a survécu à $\mathrm{S}_{64}$.

\section{Croissance en diamètre}

La Figure 5 montre des diamètres variant entre 0,42 et $0,49 \mathrm{~cm}$ pour les plants mycorhizés contre 0,41 et $0,48 \mathrm{~cm}$ pour les plants non mycorhizés. Les diamètres ont été plus élevés chez les plants mycorhizés aux doses $S_{16}$ et $S_{32}$ avec 0,49 et $0,48 \mathrm{~cm}$ contre 0,44 et 0,41 pour les plants non mycorhizés (Figure 5). Cependant, les différences de croissance en diamètre entre les traitements n'ont pas été significatives. En réponse à l'effet du sel, la mycorhization semble stimuler la croissance en diamètre au de-là de $\mathrm{S}_{16}$ au détriment de la croissance en hauteur.

\section{Chute des feuilles}

La chute cumulée des feuilles des jeunes plants stressés ou non a été plus importante lorsque ceux-ci n'ont pas été associés aux champignons endomyciorhiziens. La 
mycorhization a permis de garder davantage les feuilles sur les arbres (réduisant la restitution foliaire des sels) jusqu'à un certain seuil. En effet, à partir de $\mathrm{S}_{32}$, la différence entre les traitements s'est réduite pour s'estomper à $\mathrm{S}_{64}$ qui a été une dose létale (Figure 6).

\section{Teneur en eau des plants (\%)}

Dans les feuilles, la teneur en eau a été plus faible dans les plants associés aux champignons avec des valeurs de l'ordre de 40 à $70 \%$ que dans les témoins (50 à 75\%). Ce sont les plants témoins qui ont présenté plus d'eau (Figure 7 a). Au niveau des tiges, en dehors de $\mathrm{S}_{16}$, les traitements associés au champignon ont présenté les teneurs en eau les plus élevées avec des valeurs qui varient entre 45 et $70 \%$ (Figure 7 b). A l'exception de $\mathrm{S}_{16}$ et $\mathrm{S}_{64}$, les plants mycorhizés contiennent plus d'eau dans les racines que les témoins (Figure $7 \mathrm{c}$ ).

L'effet de l'interaction mycorhization et salinité est significatif sur la teneur en eau des racines et des tiges (Tableau 4). En milieu salin, la mycorhization jouerait donc un rôle dans l'absorption et la rétention d'eau dans les racines et leur circulation et/ou stockage dans les tiges.

\section{Teneur en sodium (Na) des plants}

La concentration en sodium dans les feuilles a été plus importante chez les plants mycorhizés aux doses $S_{16}$ et $S_{32}$ (10,3 à 12,9 $\%)$ que chez les plants non associés aux champignons (10,1 à $11,1 \%)$. Elle a été plus importante chez les plants non mycorhizés en l'absence de stress $\left(S_{0}\right)$, au seuil de réaction active au sel (dose $S_{8}$ ) et à la dose létale $S_{64}$. La teneur en sodium a été supérieure à $8 \%$ dans les feuilles quels que soient les traitements (Tableau 5).

Dans les racines, les teneurs en sodium ont été plus élevées chez les plants mycorhizés aux doses $\mathrm{S}_{8}, \mathrm{~S}_{16}$ et $\mathrm{S}_{64}(2,09$; 2,$44 ; 2,63 \%$ ) contre 1,$48 ; 1,14 ; 2,49 \%$ chez les plants non mycorhizés. L'inverse est noté à $S_{0}$ et $S_{32}$. La concentration en $\mathrm{Na}$ dans les racines a été multipliée par un facteur 3 à 6 dans les feuilles.

Tableau 1 : Composition granulométrique du substrat de croissance après 6 mois de pépinière.

\begin{tabular}{lccccc}
\hline Eléments & $\begin{array}{c}\text { Argile } \\
\mathbf{0 - 2} \boldsymbol{\mu m}\end{array}$ & $\begin{array}{c}\text { Limon fin } \\
\mathbf{2 - 2 0} \boldsymbol{\mu m}\end{array}$ & $\begin{array}{c}\text { Limon grossier } \\
\mathbf{2 0 - 5 0} \boldsymbol{\mu m}\end{array}$ & $\begin{array}{c}\text { Sable fin } \\
\mathbf{5 0 - 2 0 0} \boldsymbol{\mu m}\end{array}$ & $\begin{array}{c}\text { Sable grossier } \\
\mathbf{2 0 0} \boldsymbol{\mu m}\end{array}$ \\
\hline Teneur $(\%)$ & 7,0 & 2,4 & 1,0 & 29,2 & 60,3 \\
\hline
\end{tabular}

Tableau 2 : Evaluation finale des plantations de Atriplex lentiformis en milieu réel.

\begin{tabular}{ccccc}
\hline Année & Actions & $\begin{array}{c}\text { Quantités } \\
\text { (Nombre) }\end{array}$ & $\begin{array}{c}\text { Inventaire } \\
\text { en 2003 }\end{array}$ & $\begin{array}{c}\text { Taux de survie (\%) } \\
\text { en 2003 }\end{array}$ \\
\hline 1999 & Plantation & 2895 & 11 & 0,38 \\
2000 & Regarnis & 2854 & 133 & 4,66 \\
2001 & Regarnis & 2851 & 205 & 7,19 \\
2003 & Semis naturel & 9 & 9 & 100,00 \\
\hline
\end{tabular}


Tableau 3 : Salinité résiduelle et taux de mortalité de $A$. lentiformis.

\begin{tabular}{|c|c|c|c|c|}
\hline \multirow{2}{*}{$\begin{array}{l}\text { Doses de sel } \\
\text { g.l }{ }^{-1} \text { eau } \\
\text { d'irrigation }\end{array}$} & \multicolumn{2}{|c|}{$\begin{array}{l}\text { Salinité dans le sol à la fin de } \\
\text { l'essai en pots }\left(\text { g.l }^{-1}\right)\end{array}$} & \multicolumn{2}{|c|}{ Mortalité après 2,5 mois (\%) } \\
\hline & Avec mycorhizes & $\begin{array}{c}\text { Sans } \\
\text { mycorhize }\end{array}$ & $\begin{array}{c}\text { Avec } \\
\text { Mycorhizes }\end{array}$ & $\begin{array}{c}\text { Sans } \\
\text { Mycorhize }\end{array}$ \\
\hline$S_{0}=0$ & 0,1 & 0,4 & 0 & 0 \\
\hline$S_{8}=23,36$ & 2,2 & 1,9 & 0 & 5,56 \\
\hline$S_{16}=46,72$ & 2,5 & 3,3 & 0 & 5,56 \\
\hline$S_{32}=93,44$ & 2,8 & 3,4 & 11,1 & 11,1 \\
\hline$S_{64}=186,88$ & 3,5 & 3,6 & 100 & 100 \\
\hline
\end{tabular}

Tableau 4 : Analyse de variance sur la teneur en eau des compartiments de A. lentiformis sous stress salin.

\begin{tabular}{llccccc}
\hline Compartiments & Sources & DDL & SC & CM & F & P>F \\
\hline Feuilles & Mycor*Sel & 5 & 0,031 & 0,006 & 0,616 & 0,691 \\
R.carrée (Tiges) & Mycor*Sel & 5 & 0,143 & 0,028 & 3,125 & 0,014 \\
Racines & Mycor*Sel & 5 & 0,409 & 0,082 & 4,304 & 0,001 \\
\hline \multicolumn{2}{l}{ (DDL=Degré de liberté ; SC=somme des carrés ; CM=Carrés moyens ; P = Probabilité d'erreur } \\
Mycor*Sel = interaction mycorhization et salinité).
\end{tabular}

Tableau 5 : Teneur en $\mathrm{Na}(\%)$ des feuilles et racines à la fin de l'expérience.

\begin{tabular}{lcccccc}
\hline & \multicolumn{3}{c}{ Feuilles } & \multicolumn{3}{c}{ Racines } \\
\cline { 2 - 7 } Doses de sel $\left(\mathbf{g . l}^{-\mathbf{1}}\right.$ ) & AM & SM & AM/SM & AM & SM & AM/SM \\
\hline S0=0 & 08,79 & 11,26 & 0,78 & 1,43 & 2,03 & 0,69 \\
S8 = 23,36 & 10,00 & 11,23 & 0,89 & 2,09 & 1,48 & 1,40 \\
S16 = 46,72 & 12,95 & 11,25 & 1,15 & 2,44 & 1,14 & 2,13 \\
S32 = 93,08 & 12,29 & 10,17 & 1,20 & 1,92 & 3,20 & 0,60 \\
S64 = 186,88 & 09,65 & 11,22 & 0,86 & 2,63 & 2,49 & 1,05 \\
\hline \multicolumn{2}{c}{ (AM = avec mycorhization, SM = sans mycorhization) }
\end{tabular}
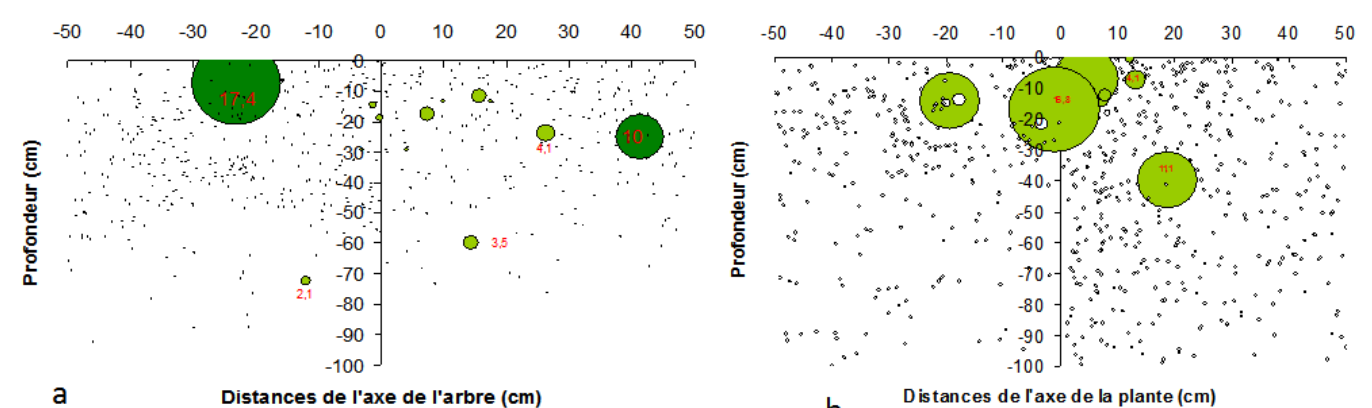

Figure 1 : Profils racinaires a: Arbre de 2000 ; b : Arbre de 2001 (les petits cercles représente les racines fines de diamètre inférieur à $1 \mathrm{~mm}$, les moyens les racines de plus de $1 \mathrm{~mm}$ et les gros les racines de plus de $1 \mathrm{~cm}$ ). 


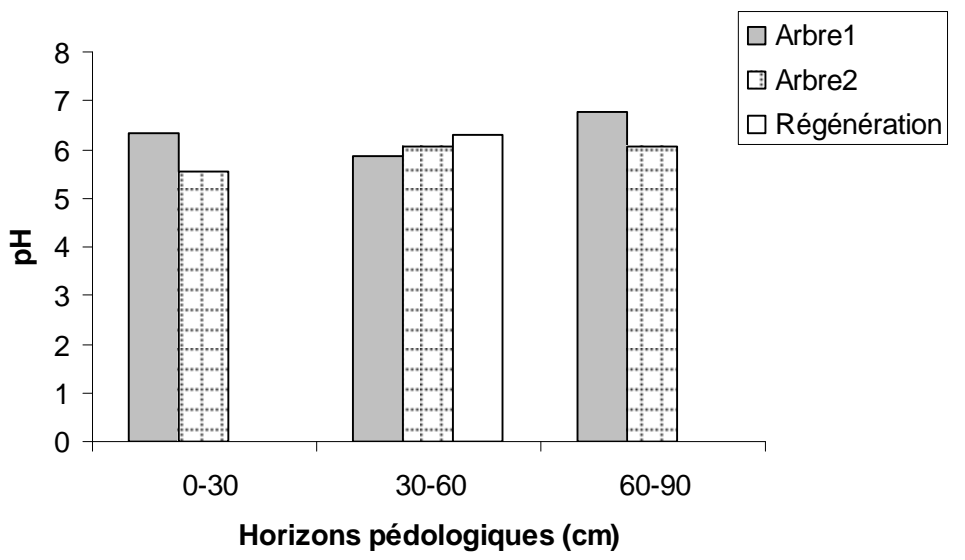

Figure 2 : Acidité du sol $(0-30 \mathrm{~cm}, 30-60 \mathrm{~cm}$ et $60-90 \mathrm{~cm})$ au pied des arbres (arbre1, arbre 2 et régénération).

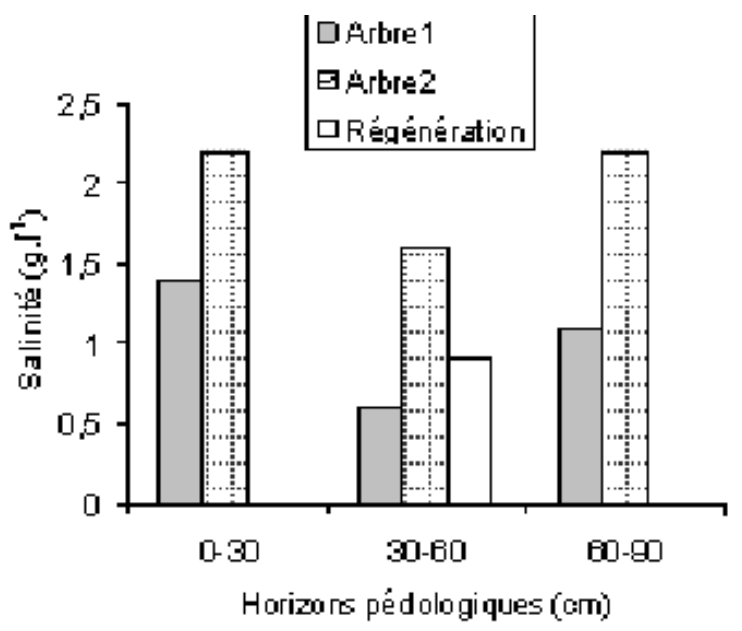

Figure 3 : Salinité autour des arbres étudiés autour des horizons 0-30 cm, 30-60 cm et $60-90 \mathrm{~cm}$.

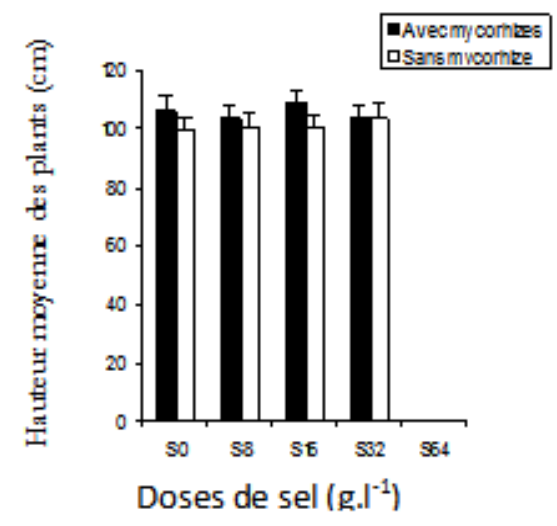

Figure 4: Influence des doses de sels (S0, S8, S16, S32 et S64) sur la hauteur des plants (mycorhizés et non mycorhizés) âgés de 13 mois. 


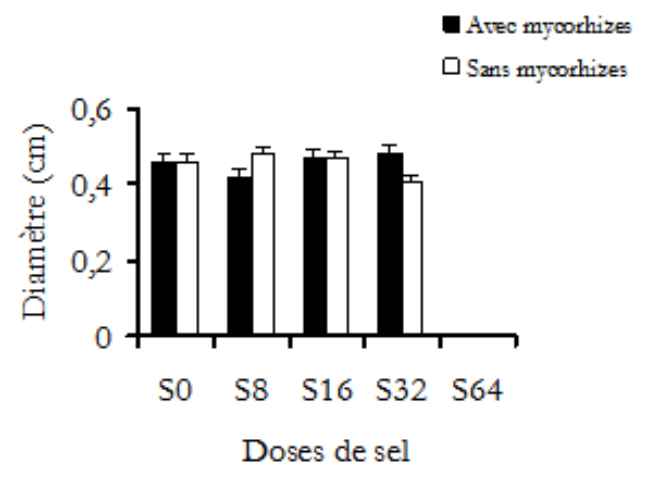

Figure 5 : Diamètre des plants de 13 mois.

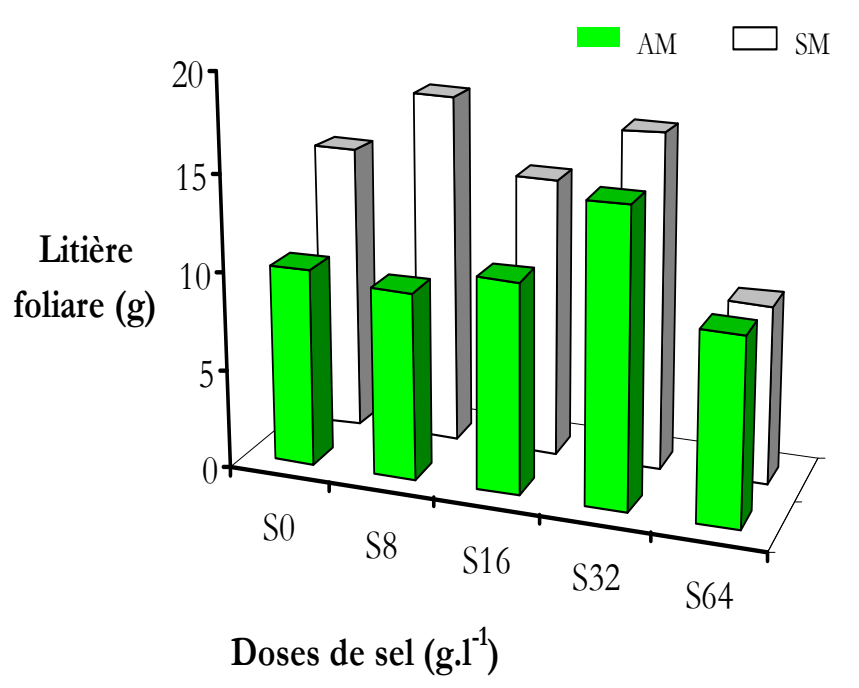

Figure 6 : Effets mycorhization sur la chute des feuilles de A. lentiformis (AM=Avec mycorhizes ; $\mathrm{SM}=$ Sans mycorhize).

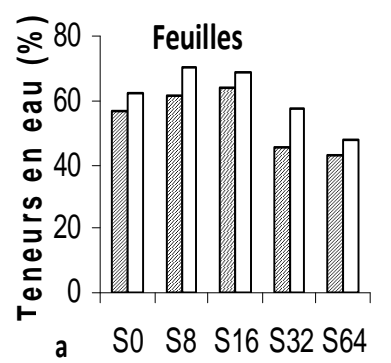

Avec Mycorhize $\square$ Sans Mycorhize
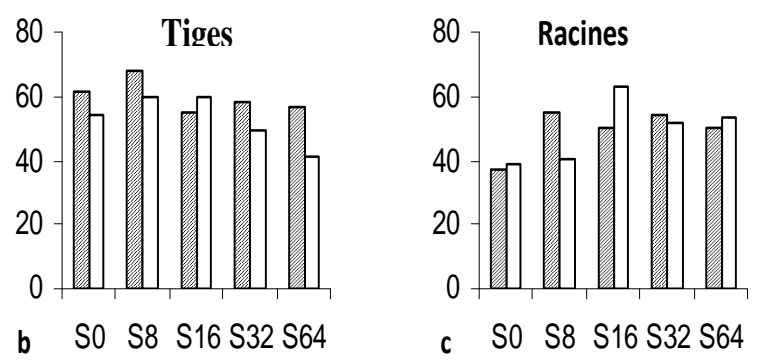

Doses de sel

Figure 7 : Teneurs en eau des plants de A. lentiformis au niveau des feuilles, racines et tiges. 


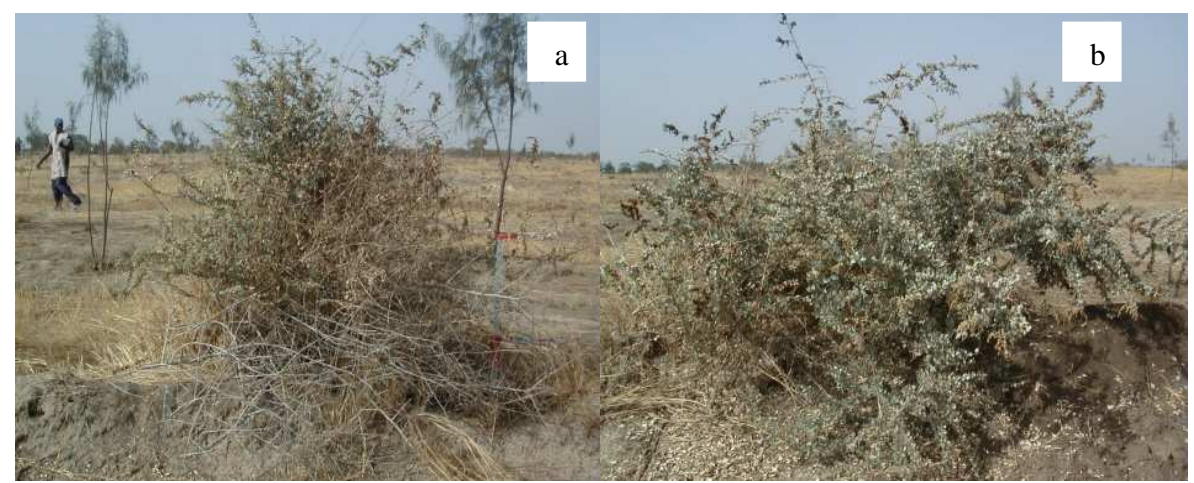

Photo 1 : Pieds 1 et 2 de A. lentiformis plantés sur des ados à sommets non amortis (a : Arbre 1; b : Arbre 2).

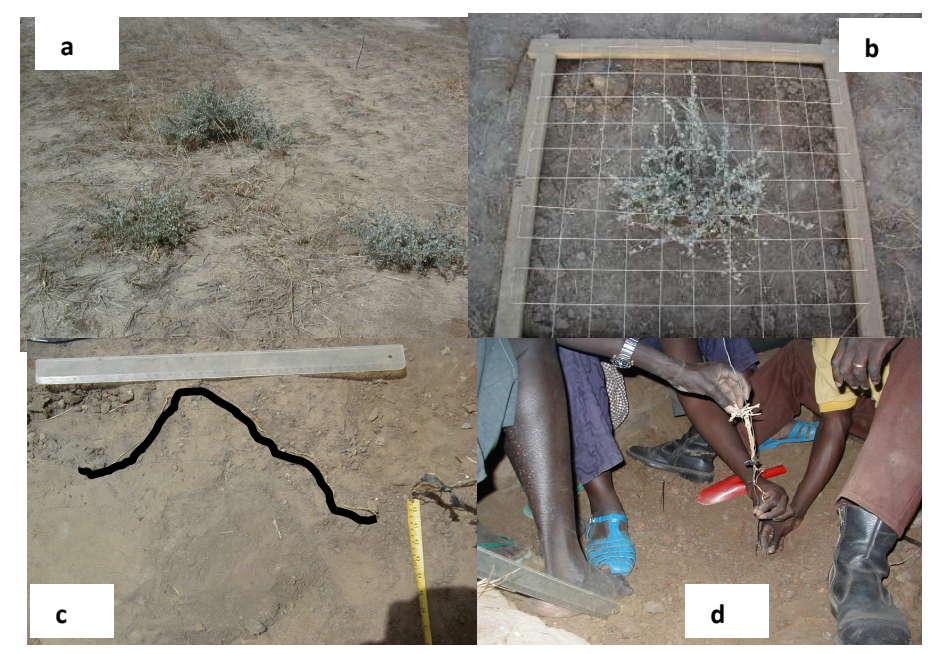

Photo 2 : Vue sur les semis naturels (a), installation de la grille de $1 \mathrm{~m} \mathrm{x} 1 \mathrm{~m}$ (b), mesures des cotes d'une racine secondaire représentée par le tracé noir (c), du pivot (d).

\section{DISCUSSION}

Les principales contraintes à l'exploitation des sols de Ndiaffate identifiées par le volet pédologie du Projet de Restauration Agronomique des Sols Salés (PRASS) sont l'acidité, la salinité et la compacité en saison sèche, notamment des sols situés en aval de la toposéquence (Anonyme, 2004). L'évaluation in situ du comportement de Atriplex lentiformis a montré un très fort taux de mortalité qui semble lié à ces contraintes. Cependant, un phénomène important a été noté dans le site de Ndiaffate : la reproduction séminale de Atriplex lentiformis. Un total de 7 semis naturels ont été inventoriés aussi bien en basse qu'en moyenne terrasses, ce qui prouve que l'espèce peut bien s'adapter dans le milieu.

En termes de conductivité électrique, la salinité a été basse selon les normes sénégalaises qui estiment un sol salé à partir de $4 \mathrm{mS} . \mathrm{cm}^{-1}$ de conductivité ou 2,44 g. $\mathrm{l}^{-1}$ (Anonyme, 1992). Ce seuil a été variable selon les espèces. En effet, en Australie comme ailleurs, Sun et Dickinson (1995) considèrent les sols hautement salés au-dessus 
de $1,1 \mathrm{mS} . \mathrm{cm}^{-1}$ pour des espèces des genres Eucalyptus et Casuarina.

Globalement, la situation n'est que très légèrement salée en juin selon les normes sénégalaises établies en 1992. Mais quelle que soit la plante considérée, la salinité a été plus forte en surface $(0-30 \mathrm{~cm})$ et en profondeur $(60-90 \mathrm{~cm})$ que par rapport à l'horizon intermédiaire $(30-60 \mathrm{~cm})$. Cela pourrait être lié à la remontée capillaire des sels dans les couches profondes et à leur accumulation en surface du fait de la forte évaporation et des dépôts éoliens. A l'horizon $30-60 \mathrm{~cm}$ de la plante 1 et des régénérations, la conductivité électrique était de l'ordre de $1 \mathrm{mS} . \mathrm{cm}^{-1}$.

Ainsi, autour de la plante 1, pour laquelle la salinité et l'acidité ont été relativement plus basses, les plus grosses racines se répartissent dans les 70 premiers $\mathrm{cm}$ $\mathrm{du}$ sol. Autour de la plante 2, où la salinité $\mathrm{a}$ été plus élevée, les plus grosses racines ont été dans les 40 premiers $\mathrm{cm}$. Cependant, les racines fines sont réparties dans tout le profil de façon plus homogène chez la plante 2 .

Concernant la compacité en saison sèche, l'hypothèse de l'enracinement traçant serait reliée uniquement à la plantation sur ados. Ce résultat montre que par semis naturel, on obtient à la fois un pivot puissant qui traverse les couches de sol riches en jarosite, compactées par les argiles avec la présence de concrétions ferrugineuses. Le problème de l'enracinement normal de $A$. lentiformis à Ndiaffate serait donc surtout lié au choix de la technique d'installation.

Un autre phénomène important à signaler a été la différence des systèmes racinaires des individus issus de plantation artificielle sur des ados et des individus issus de régénération naturelle. Ces derniers, contrairement aux premiers, développent un système racinaire pivotant, non ramifié mais contournant les obstacles en formant parfois des angles successifs de $90^{\circ}$ avec la verticale. Ce pivot parvient à traverser les concrétions ferrugineuses. Par contre, sur les ados, les plants installés ont tendance à maintenir leur système racinaire dans les horizons de surface, rendus meubles par la confection des diguettes.

Avec les résultats de cette évaluation in situ, on peut déjà retenir que la contrainte liée à la compacité des sols peut être réduite avec l'amélioration des techniques d'installation par semis directs ou aplatissement des ados sur les diguettes avant plantation ou alors par association semis direct et plantation.

Le rapport final du projet PRASS signale que l'un des résultats les plus importants obtenus après caractérisation des sols de la parcelle expérimentale de Ndiaffate est que le phénomène d'acidité des sols peut être efficacement combattu avec la technique du chaulage (Anonyme, 2004). Quant à la salinité, la même source indique que sa neutralisation est complexe. Elle indique aussi la nécessité d'analyser la nature des sels accumulés aussi bien au niveau des sols que des plantes.

Pour l'évaluation de l'effet de la salinité ex situ, l'accent a été mis sur le renforcement des capacités intrinsèques de résistance (Epstein et al., 1980; Conrad, 1987) de Atriplex lentiformis par association mycorhizienne. Sur le terrain, les résultats montrent que la salinité a été plus importante en surface $(0-30 \mathrm{~cm})$ et en-dessous de $60 \mathrm{~cm}$ $(60-90 \mathrm{~cm})$ que dans les horizons intermédiaires $(30-60 \mathrm{~cm})$ autour des plantes étudiées. Cela pourrait, en plus de la compacité, expliquer pourquoi les arbres gardent leurs grosses racines au-dessus de 60 $\mathrm{cm}(0-50 \mathrm{~cm})$ puisqu'il est connu que $A$. lentiformis croît habituellement sur des sols à pH plutôt basique (Van Epps et al., 1982). Au laboratoire, les plants mycorhizés ou non, ont été arrosés avec de l'eau hautement chargée en sels de sodium à des doses supérieures à la concentration de l'eau de mer qui est de $35 \mathrm{~g} . \mathrm{l}^{-1}$. A. lentiformis peut supporter pendant plus d'un mois plus de 46 g..$^{-1}$ de l'eau d'arrosage avec seulement $5 \%$ de mortalité quand les plants ne sont pas mycorhizés. La mycorhization permet de sauver tous les plants malgré la forte dose de sel appliquée. Et même à 93 g. $1^{-1}$, la mortalité n'est que de $11 \%$. 
Mais, au-delà de cette dose, la mycorhisation n'a plus d'effets sur la survie des plants. Malgré une croissance plus élevée des plants mycorhizés, leur teneur en eau dans les feuilles a été plus basse que celle des plants non mycorhizés. Des résultats semblables ont été obtenus chez Triticum durum Desf., variété de blé dur alégrien où $80,35 \%$ d'eau a été obtenu avec une dose de $\mathrm{NaCl}$ de 4 g. l $^{-1}$ avec un potentiel hydrique de -1,41 MPA (au stade 4 feuilles) et une hydratation foliaire de $70,22 \%$ pour la même concentration en sel (Chorfi, 2009). Selon ce dernier auteur, le stress salin induit une baisse du contenu relatif en eau. L'explication est donnée par Mâalem (2011) qui a observé des signes visibles de l'impact du stress salin essentiellement sur les feuilles de Atriplex halimus, A. nummularia et A. canescens. Cet impact s'est manifesté par la présence de caractères anatomiques adaptatifs caractéristiques des halophytes dont les plus importants concernent l'épiderme à travers une polystratification qui agit manifestement contre la perte de l'eau, des feuilles vers l'extérieur. Ainsi, elles paraissent, plus au moins, succulentes avec des stomates enfuies dans de profondes chambres sus-stomatique. Pour la teneur en sodium des feuilles, la mycorhization a coïncidé avec des teneurs plus basses respectivement aux plus faibles et forts stress salins $\left(23,36\right.$ et de 186,88 g. l $\left.^{-1}\right)$. Ceci s'était traduit par une plus faible chute des feuilles à ces doses. Par contre, aux doses de 46,72 et de 93,44 g. l $^{-1}$, les teneurs en sodium dans les plants mycorhizés (rapport 1,5 à 1,20) et la chute des feuilles ont été plus élevées. Donc, les plants mycorhizés utilisent plus de sel d'arrosage que les non mycorhizés pour justifier la différence de salinité résiduelle du sol plus faible en présence du champignon mycorhizien essentiellement aux doses 46,72 et 93,44 g. $1^{-1}$. Cependant, la mycorhization limite globalement la perte foliaire comparativement aux plants mycorhizés.

Au niveau des racines et des tiges, la mycorhization jouerait un rôle dans l'absorption et la circulation de l'eau puisque les différences ne sont pas significatives. Ce rôle serait lié à la concentration en sel car dans les racines des plants mycorhizés, en dehors de la dose de 93,44 g.1 ${ }^{-1}$, il y a plus de $\mathrm{Na}^{+}$dans les racines des plants mycorhizés (rapport 1,05 à 2,13).

A. lentiformis est très résistant à la salure (93 g..$^{-1}$ avec $11 \%$ de mortalité). La mycorhization, par ses effets positifs sur la croissance et le maintien des feuilles, renforce cette résistance qui se traduit par une survie importante des plants malgré des teneurs en eau foliaire et racinaire faibles et une teneur en sodium importante.

Ainsi, l'analyse combinée des teneurs en eau et en sodium des systèmes aériens et souterrains, a permis de confirmer le stockage de sodium dans les parties aériennes. Ce stockage de sodium dans les feuilles augmente (jusqu'à 12\%) au-delà du seuil de réaction active au sel $\left(23,36\right.$ g. . $\left.^{-1}\right)$ et se renforce dans les parties aériennes par la mycorhization. La capacité à stocker le sel dans les parties aériennes a été déterminante dans le niveau de tolérance au sel des espèces (Levingeron et al., 1995 ; Shannon, 1997). Lorsque cette stratégie s'accompagne de la réduction des chutes foliaires chez les plants mycorhizés, il a été noté une faible toxicité saline. Les teneurs en sodium 3 à 6 fois plus élevées dans les feuilles que dans les racines, montrent que A. lentiformis développe d'abord la faculté d'accumulation et/ou d'excrétion de sel au niveau des parties aériennes. Ce phénomène déjà signalé par Epstein et al. (1980), renforce l'assertion de Conrad (1987) qui indiquait que A. lentiformis stocke le sel dans les feuilles.

A l'intérieur des feuilles, le sel est stocké dans l'apoplasme ou espace intercellulaire ou alors réexporté vers les racines selon Levigneron et al. (1995). L'augmentation du sodium dans les racines de A. lentiformis pourrait être la résultante de la recirculation du sel. En effet, en regardant le taux de sodium des plants mycorhizés à partir du seuil de réaction au sel $\left(23,36\right.$ g. $\left.l^{-1}\right)$, il ressort qu'il pourrait y avoir une redistribution du sodium vers les racines favorisée par la 
mycorhization. Cette redistribution correspond à un gain de tolérance d'après Levigneron et al. (1995). Dans ce cas ce sont les cellules racinaires qui assurent la protection des parties aériennes en limitant la quantité de sodium accumulée dans les cellules de ces dernières. Blits et Gallangher (1990) ont déjà montré l'augmentation de la teneur en $\mathrm{Na}^{+}$des racines de Kosteletzkya virginica avec la hausse de $\mathrm{NaCl}$ dans le milieu extérieur. Chez Atriplex lentiformis, les teneurs en sodium des racines sont inférieures à $4 \%$, largement en-dessous de celles des feuilles qui peuvent atteindre $12 \%$ chez les plants mycorhizés.

\section{Conclusion}

Il ressort de cette étude que la salinité et l'acidité, de même que la compacité du sol de Ndiaffate affectent les profils aériens et souterrains des plantes. Mais les résultats des recherches menées aux plans physicochimique des sols et biologique montrent que le problème de $A$.lentiformis dans le site de Ndiaffate ne serait pas la salinité à laquelle il développe des stratégies de résistance. Cette résistance est significativement améliorée par la mycorhization avec Rhizophagus irregulare. Il ne s'agit pas non plus de l'acidité qui peut être combattue par le chaulage. Des trois contraintes initiales considérées, il ne reste plus que la compacité face à laquelle la plante peut lutter si on améliore les techniques d'installation par l'association du semis direct et de la plantation sur des ados aplatis ou sur le versant. Autrement dit, A. lentiformis devrait bien convenir dans les conditions des sols de Ndiaffate si les mesures techniques nécessaires sont prises. Ces mesures peuvent être renforcées par l'amélioration biologique des jeunes plants.

\section{REMERCIEMENTS}

Les auteurs remercient Feu Roger Pontanier, Dr Robin Duponnois et Dr Dominique Masse de l'IRD pour leur appui technique et scientifique.

\section{REFERENCES}

Anonyme. 1992. Qualité des sols : méthodes techniques de conservation et de régénération des sols. Normes sénégalaises. Institut Sénégalais de Normalisation (ISN), NS 05-049, p. 20.

Anonyme. 2004. Rapport technique final. Projet de Restauration Agronomique des Sols Salés. Africare, ISRA, ISE/UCAD, Ipalac, Dakar, p. 61.

Blits KC, Gallagher JL. 1990. Salinity tolerance of Kosteletzkya virginica.II. Root growth, lipid content, ion and water relations. Plant, Cell and Environment, 13: $419-425$.

Chatterton NJ, McKell CM. 1969. Atriplex polycarpa: I. Germination and growth as affected by sodium chloride in water cultures. Agron. J., 61: 448-450.

Chorfi A. 2009. Contribution à l'étude de la résistance à la salinité chez une variété de blé dur algérien (Triticum durum Dsf.) var Mohamed Ben Bachir. Sciences et Technologies, 29: 41-44.

Conrad CE. 1987. Common shrubs of chaparral and associated ecosystems of southern California. Gen. Tech. Rep. PSW-99. Berkeley, CA: U.S. Department of Agriculture, Forest Service, Pacific Southwest Forest and Range Experiment Station, p. 86.

Epstein E, Norlyn JD, Rush D, Kingsbury R, Kelley G, Cunningham G, Wrona A. 1980. Saline culture of crops- a genetic approach. Science, 210(4468): 399-404.

Franclet E, Le Houérou HN. 1971. Les Atriplex en Tunisie et en Afrique du Nord. FAO : Rome ; 249.

Land Degradation Assesment (LADA). 2003. L'évaluation de la dégradation des terres au Sénégal. Rapport préliminaire, p. 59.

Le Houérou HN. 1992. The role of saltbushes (Atriplex spp.) in arid land rehabilitation in the Mediterranean Basin : a review. Agroforestry Systems, 18(2): 107-148.

Levigneron A, Lopez F, Vansuyt G, Berthomieu, Fourcroy P, Casse-Delbart 
F. 1995. Les plantes face au stress salin. Cahiers Agricultures, 4(4): 263-273.

Mâalem S. 2011. Etude de l'interaction entre le phosphore et le chlorure de sodium sur trois espèces végétales halophytes du genre Atriplex (A. halimus, A. canescens et A. nummularia). Thèse de Doctorat, Université Badji Mokhtar Annaba, p. 169.

Olivares AY, Johnston M. 1978. Alternativas de mejoramiento en la emergencia de Atriplex repanda. Phil. Phyton, 36(2): 129-137.

Orev Y. 1987. Manuel pratique pour l'amélioration des pâturages en zones semi-arides. Rapport technique, CTA. Genève, p.123.

Phillips JM, Hayman DS. 1970. Improved procedure for clearing roots ans staining parasitic and vesicular-arbuscular mycorrhizal fungi for rapid assessment of infection. Trans. Brit. Mycol. Soc., 55(1): 158-161.
Roussel J. 1995. Pépinières et Plantations forestières en Afrique tropicale sèche. ISRA/CIRAD, Unival, Dakar, p. 435.

Rusbridge S, Bradley G, Bradley G. 1996. Plant identification hand book for land rehabilitation in the goldfields of western Australia. Goldfields Land Rehabilitation, p. 114.

Sun D, Dickninson GRD. 1995. Salinity effects on tree growth, roots distribution and transpiration of Casuarina cunninghamiana and Eucalyptus camaldulensis planted on a saline soil in tropical north Australia. For. Ecol. Manage, 77(1-3): 127-138.

Shannon MC. 1997. Adaptation of plants to salinity. Advances in Agronomy, 60: 75120.

Van Epps GA, Barker JR, McKell CM. 1982. Energy biomass from large rangeland shrubs of the Intermountain United States. Journal of Range Management, 35(1): 22-25. 